\title{
HL7 Terminology Management for Disease Surveillance
}

\author{
Emily Roberts*, Theron Jeppson, Rachelle Boulton and Josh Ridderhoff
}

Informatics Program, Utah Department of Health, Salt Lake City, UT, USA

\section{Objective}

The objective of this abstract is to illustrate how the Utah Department of Health processes a high volume of electronic data. We do this by translating what reporters send within an HL7 message into "epidemiologist" language for consumption into our disease surveillance system.

\section{Introduction}

In 2013, the Utah Department of Health (UDOH) began working with hospital and reference laboratories to implement electronic laboratory reporting (ELR) of reportable communicable disease data. Laboratories utilize HL7 message structure and standard terminologies such as LOINC and SNOMED to send data to UDOH. These messages must be evaluated for validity, translated, and entered into Utah's communicable disease surveillance system (UT-NEDSS), where they can be accessed by local and state investigators and epidemiologists. Despite the development and use of standardized terminologies, reporters may use different, outdated versions of these terminologies, may not use the appropriate codes, or may send local, home-grown terminologies. These variations cause problems when trying to interpret test results and automate data processing. UDOH has developed a two-step translation process that allows us to first standardize and clean incoming messages, and then translate them for consumption by UT-NEDSS. These processes allow us to efficiently manage several different terminologies and helps to standardize incoming data, maintain data quality, and streamline the data entry process.

\section{Methods}

UDOH uses the Electronic Message Staging Area (EMSA) to receive ELR messages, manage terminologies such as LOINC and SNOMED, translate messages, and automatically enter laboratory data into UT-NEDSS. LOINCs and other terms, such as facility name, sent by reporting facilities in an HL7 message are considered child terms. All child terms are mapped to a master LOINC or term and each master LOINC or term is mapped to a specific value within UTNEDSS. In EMSA, the rules engine used for automated processing of electronic data is set to run at the master level and these rules will determine how the message is processed. No rules are set up or run on child terms.

\section{Results}

As of 09/20/2017, EMSA contains 2,613 unique child LOINCs that are mapped to 906 master LOINCs. Those 906 master LOINCs are mapped to 179 UT-NEDSS test types and 2003 child facility names are mapped to 1043 master facility names.

\section{Conclusions}

Mapping child terminologies from an HL7 message to a master vocabulary helps us to standardize incoming data, allows us to accept non-standard terminologies and correct reporting errors. Translating this data into a format that is understandable to epidemiologists and investigators enables UT-NEDSS to work effectively in identifying outbreaks and improving health outcomes. This framework is working for ELR and will continue to grow and accept more data and the different terminologies that come with that.

Keywords

HL7; Terminology; Surveillance; Translate

\section{*Emily Roberts}

E-mail: erroberts@utah.gov 